\title{
INVARIANTS INTÉGRAUX FONCTIONNELS POUR DES ÉQUATIONS AUX DÉRIVÉES PARTIELLES D'ORIGINE GÉOMÉTRIQUE
}

\author{
JEAN PIERRE BOURGUIGNON \\ Centre de Mathématiques, URA-CNRS No. 169, Ecole Polytechnique \\ F-91128 Palaiseau Cedex, France
}

Le maintenant classique problème de Nirenberg s'énonce : "étant donnée une fonction $f$ sur la sphère à deux dimensions, existe-t-il une métrique $g$ conforme à la métrique standard $c$ dont la courbure de Gauss $s_{g}$ soit $f$ ?" Pour que ce problème ait une solution, on voit facilement qu'il est nécessaire que la fonction $f$ soit positive quelque part sur la sphère à cause du théorème de Gauss-Bonnet, selon lequel l'intégrale $\int_{S^{2}} s_{g} v_{g}$ (où $v_{g}$ désigne l'élément de volume de la métrique $g$ ) ne dépend pas de la métrique et vaut $4 \pi$. Dans [5], J. L. Kazdan et F. Warner donnent d'autres conditions nécessaires sur la fonction $f$ pour que le problème ait une solution, conditions qui peuvent s'exprimer ainsi : "Si $\xi$ est une première harmonique sphérique, alors

$$
\int_{S^{2}}\left(\nabla^{c} \xi, \nabla^{c} s_{g}\right) v_{g}=0
$$

où $\nabla^{c}$ désigne le gradient dans la métrique $c$ ".

La preuve initiale qu'ils en ont donnée se fait par un calcul explicite utilisant le fait que les premières harmoniques sphériques sont restrictions à la sphère $S^{2}$ des fonctions linéaires sur $\mathbb{R}^{3}$. Ces relations fournissent des exemples non triviaux de fonctions qui ne peuvent être la courbure scalaire d'une métrique conforme à $c$ (on dit qu'elles sont "interdites") tels les fonctions dépendant de façon monotone de la distance à un point.

Si on remplace la courbure de Gauss par la courbure scalaire, le problème de Nirenberg garde un sens en dimension supérieure, et pour le problème ainsi étendu les obstructions précitées demeurent. (Pour des développements récents sur ce sujet, on peut consulter [1] et [3].) 
Dans cet exposé nous nous proposons de montrer que ces identités ne sont en rien attachées à la sphère, mais sont en fait universelles. Dans un cadre approprié que nous décrivons dans la Section 1, leur démonstration devient alors élémentaire. De plus on peut obtenir de nouvelles identités dont nous donnons des exemples dans la Section 2. Il devient alors possible de relier précisément ce problème avec d'autres comme la recherche de métrique d'Einstein sur les variétés kählériennes à première classe de Chern positive pour laquelle A. Futaki a donné dans [4] des obstructions qui ressemblent étrangement à celles de Kazdan-Warner. Nous abordons ce point dans la Section 3. Nous concluons par quelques remarques sur le lien de notre approche avec la théorie des anomalies qui intéressent beaucoup les physiciens théoriciens en ce moment.

1. Le cadre géométrique. Nous allons travailler sur un espace $\mathcal{O}$ d'objets géométriques, sur lequel un groupe de Lie $G$ agit. Nous allons concentrer notre attention sur certaines formes différentielles fermées $\alpha$ sur $\mathcal{O}$ invariantes par l'action du groupe $G$. Une application élémentaire de la théorie des invariants intégraux d'Elie Cartan nous amène à faire les constatations suivantes : à cause de l'invariance de la forme différentielle $\alpha$, pour tout élément $X$ de l'algèbre de Lie $\mathfrak{g}, \mathcal{L}_{X} \alpha=0$; par ailleurs, d'après la relation fondamentale $\mathcal{L}_{X}=i_{X} \circ d+d \circ i_{X}$ appliquée à la forme fermée $\alpha$ et à un élément $X$ de l'algèbre de Lie, on voit que $d i_{X} \alpha=0$, autrement dit que la fonction $i_{X} \alpha$ est constante.

Nous affirmons que les relations d'intégrabilité de Kazdan-Warner ainsi que celles d'A. Futaki proviennent toutes de l'application de ce calcul élémentaire après qu'on ait choisi convenablement l'espace des objets géométriques, le groupe qui agit sur lui et les formes différentielles fermées à considérer.

Nous expliquons ces choix dans le cas du problème de Nirenberg généralisé. Nous partons d'une variété différentielle compact $M$ de dimension $n$. Nous fixons une classe conforme $\Gamma$ de métriques riemanniennes sur $M$, autrement dit nous considérons toutes les métriques $g$ qui se déduisent de l'une d'entre elles $g_{0}$ par multiplication par une fonction positive $e^{2 f}$. (Lorsque $n \geq 3$, il est classique, et pratique, d'écrire $e^{2 f}$ sous la forme $u^{4 n /(n-2)}$ en supposant $\left.u>0\right)$. Ce sera notre espace $\mathcal{O}$ d'objets géométriques.

Le groupe de Lie $G$ sera le groupe des transformations conformes de la variété conforme $(M, \Gamma)$ que nous faisons opérer naturellement sur $\Gamma$ comme suit : pour $\varphi$ dans $G$ et $g$ dans $\Gamma$, nous posons $\varphi(g)=\varphi^{*} g$ où dans le membre de droite les objets prennent le sens qu'ils ont habituellement en dimension finie. Si $X$ est un élément de l'algèbre de Lie $\mathfrak{g}$ de $G$ (autrement dit un champ de vecteurs conforme), le champ de vecteurs $\bar{X}$ qu'il induit sur $\mathcal{O}$ est donc défini par $\bar{X}(v)=\mathcal{L}_{X} v$.

Il sera commode d'identifier $\Gamma$ à l'ensemble des éléments de volume définis sur $M$, une métrique $g$ étant représentée par son élément de volume $v_{g}$ (à cause de cette identification, si $g=e^{2 f} g_{0}$, alors $\left.v_{g}=e^{n f} v_{g_{0}}\right)$. Grâce à cette identification, on peut voir l'espace tangent à $\Gamma$ en un point $g$ identifié à $v_{g}$ comme l'ensemble des $n$-formes différentielles sur $M$ (pour simplifier la présentation, nous supposons 
$M$ orientable), et par suite l'espace cotangent $T^{*} \Gamma$ apparaît comme l'espace des fonctions sur $\Gamma$. Une forme différentielle sur $\Gamma$ associe donc à une métrique $g$ de la classe conforme $\Gamma$ une fonction sur $M$. Dans le cadre de notre problème, la forme différentielle à considérer est la forme $\sigma$ qui à $g$ associe sa courbure scalaire, donc définie par $\sigma(g)=s_{g}$.

Lemme 1. Sur $\Gamma$, la forme différentielle $\sigma$ est fermée.

P r e u ve. Pour vérifier que $\sigma$ est fermée, il suffit d'évaluer $d \sigma$ sur deux vecteurs tangents $V_{1}$ et $V_{2}$ en un point $g$ de $\Gamma$. Comme $\Gamma$ a été identifié à un ouvert de l'espace vectoriel des $n$-formes différentielles sur $M$, on peut supposer que $V_{1}$ et $V_{2}$ ont été étendus au voisinage de $g$ comme des champs de vecteurs constants. Par application de la formule donnant le cobord d'une forme différentielle, nous avons donc

$$
\begin{aligned}
d \sigma\left(V_{1}, V_{2}\right) & =V_{1} \cdot \sigma\left(V_{2}\right)-V_{2} \cdot \sigma\left(V_{1}\right) \\
& =\left.\frac{d}{d t}\left(\int_{M} s_{g+2 t f_{1}} V_{2}\right)\right|_{t=0}-\left.\frac{d}{d t}\left(\int_{M} s_{g+2 t f_{2}} V_{1}\right)\right|_{t=0}
\end{aligned}
$$

où nous avons utilisé le fait que la courbe de métriques $\left(1+\operatorname{tn} f_{i}\right) g$ a pour vecteurvitesse $V_{i}=n f_{i} v_{g}(i=1,2)$ dans la représentation que nous avons choisie. Comme on peut permuter $\int_{M}$ et $d / d t$ et que la linéarisation de la courbure scalaire est donnée par la formule

$$
\left.\frac{d}{d t}\left(s_{g+2 t f_{i}}\right)\right|_{t=0}=2(n-1) \Delta_{g} f_{i}-2 s_{g} f_{i}
$$

(où $\Delta_{g}$ désigne le laplacien de la métrique $g$ ), nous obtenons donc

$$
\begin{aligned}
d \sigma\left(V_{1}, V_{2}\right)= & \int_{M}\left[2(n-1) \Delta_{g} f_{1}-2 s_{g} f_{1}\right] n f_{2} v_{g} \\
& -\int_{M}\left[2(n-1) \Delta_{g} f_{2}-2 s_{g} f_{2}\right] n f_{1} v_{g} \\
= & \int_{M} 2 n(n-1)\left[\left(\Delta_{g} f_{1}\right) f_{2}-\left(\Delta_{g} f_{2}\right) f_{1}\right] v_{g}=0,
\end{aligned}
$$

car $\Delta_{g}$ est auto-adjoint.

Il nous faut maintenant démontrer la naturalité.

LEMME 2. Sur $\Gamma$, la forme différentielle $\sigma$ est invariante par le groupe $G$ des transformations conformes de $\Gamma$.

Preuve. Pour cela, pour un élément $\varphi$ du groupe $G$, nous évaluons la forme 
$\varphi^{*}(\sigma)$ au point $v=v_{g}$ sur le vecteur tangent $V$. Nous avons

$$
\begin{aligned}
\varphi^{*}(\sigma)_{v}(V) & =\sigma_{\varphi^{*} v}(T \varphi(V))=\int_{M} s_{\varphi^{*} g} \varphi^{*} V \\
& =\int_{M}\left(s_{g} \circ \varphi\right) \varphi^{*} V=\int_{M} s_{g} V=s(V) .
\end{aligned}
$$

Dans ce calcul, il faut prendre garde que nous avons utilisé la même notation pour l'image réciproque des formes sur la variété de dimension finie $M$ et sur la variété $\mathcal{O}=\Gamma$ de dimension infinie. Les seules propriétés utilisées sont la naturalité de la courbure $\left(s_{\varphi^{*} g}=s_{g} \circ \varphi\right)$ et le théorème de changement de variable dans les intégrales sur une variété sans bord.

De la discussion que nous avons présentée au début de ce paragraphe, il ressort que, pour tout champ de vecteurs conforme $X$, la fonction $i_{\bar{X}} \sigma$ est constante, ce qui nous conduit directement à la relation de Kazdan-Warner comme l'établit la proposition suivante.

Proposition 3. Soit $(M, \Gamma)$ une variété conforme et $G$ son groupe de transformations conformes. Pour tout champ de vecteurs conforme $X$ et pour toute métrique $g$ dans la classe conforme $\Gamma$,

$$
\int_{M} X \cdot s_{g} v_{g}=0
$$

Preuve. Si nous traduisons la constance de la fonction $i_{\bar{X}} \sigma$, nous obtenons pour toutes métriques $g$ et $g^{\prime}$ de $\Gamma$,

$$
\int_{M} s_{g} \mathcal{L}_{X} v_{g}=\int_{M} s_{g^{\prime}} \mathcal{L}_{X} v_{g^{\prime}},
$$

ce qui par intégration par partie donne

$$
\int_{M} X \cdot s_{g} v_{g}=\int_{M} X \cdot s_{g^{\prime}} v_{g^{\prime}}
$$

Il ne nous reste donc plus qu'à démontrer que cette intégrale est nulle. Ce fait est spécial au groupe des transformations conformes d'une variété compacte. En effet, si $G$ est un groupe compact, pour évaluer l'intégrale on peut prendre une métrique dans la classe conforme obtenue par moyenne sur le groupe compact $G$, donc telle que $G$ soit pour elle un groupe d'isométries. Par suite la courbure scalaire de cette métrique est invariante par l'action de $G$, donc en particulier annihilée par les champs de vecteurs $X$ de $\mathfrak{g}$. Si le groupe $G$ est non compact, alors par un théorème dû à M. Obata (cf. [7]) et à J. LelongFerrand (cf. [6]), la classe conforme $\Gamma$ est nécessairement la classe standard sur la sphère. Il suffit alors de prendre une métrique à courbure constante pour évaluer l'intégrale. 
Une autre preuve de cette relation est donnée dans [2]. Pour $n \geq 3$, elle est obtenue directement par intégration sur la variété $M$ de l'identité que satisfait un champ de vecteurs conforme $X$ pour toute métrique $g$ de la classe conforme $\Gamma$, à savoir

$$
\Delta_{g}\left(\operatorname{div}_{g} X\right)=\frac{1}{n-1} s_{g} \operatorname{div}_{g} X+\frac{n}{2(n-1)} X \cdot s_{g}
$$

Le cas $n=2$ nécessite une analyse plus fine, et met en jeu la définition de deux actions du groupe conforme sur l'espace des fonctions (cf. [2]).

2. De nouvelles relations intégrales. Dans ce paragraphe, nous donnons de nouveaux exemples de relations intégrales obtenu comme invariants intégraux fonctionnels par le schéma que nous avons décrit dans la Section 1.

D'abord il va de soi que pour toute fonction $F$ définie sur l'espace $\mathcal{O}$ d'objets géométriques (i.e. la classe conforme $\Gamma$ dans le cas qui nous occupe) invariante par le groupe $G$, sa différentielle $d F$ donne naissance à un invariant intégral fonctionnel. Cela donne une infinité d'exemples qui sont a priori tautologiques mais qui se révèlent être cependant intéressants. Donnons-en quelques exemples dans le cas particulier considéré pour illustrer notre propos.

La 1-forme différentielle sur $\Gamma$ considérée dans la Section précédente est en fait une différentielle exacte pour $n \geq 3$, car on a alors $\sigma=d \Sigma$ avec $\Sigma=\frac{n-2}{n} \int_{M} s_{g} v_{g}$. Cela "explique" pourquoi la preuve donnée dans [2] se simplifie directement dans ce cas-là.

Parmi les fonctions $F$ géométriques, il est naturel de considérer la famille $\Sigma_{k}$ pour $k \in \mathbb{N}$ définie par

$$
\Sigma_{k}\left(v_{g}\right)=\int_{M} s_{g}^{k} v_{g}
$$

de telle sorte que $\Sigma_{1}=\Sigma$. Par un calcul direct, on trouve que

$$
d \Sigma_{k}=(n-2) k \Delta\left(s^{k-1}\right)+(n-2 k) s^{k},
$$

où on rappelle qu'il faut interpréter le membre de droite comme une 1-forme différentielle sur $\Gamma$. Quelques cas particuliers méritent d'être mentionnés : pour $n=4$ par exemple, on voit que la 1 -forme différentielle $v_{g} \mapsto \Delta_{g} s_{g}$ est exacte. Il lui est donc associée une loi de conservation qui s'énonce comme suit :

Proposition 4. Pour tout champ de vecteurs conforme $X$ et pour toute métrique $g$ d'une classe conforme $\Gamma$ définie sur la variété $M$ de dimension 4 ,

$$
\int_{M} X \cdot \Delta_{g} s_{g} v_{g}=0 .
$$

Un énoncé analogue peut bien sûr être donné pour une variété de dimension $2 k$ en remplaçant $s$ par $s^{k-1}$.

Un autre exemple d'invariant intégral fonctionnel qui se déduit de la considération de la différentielle d'une fonction invariante est le suivant. Soit $\lambda_{1}$ la 
première valeur propre de l'opérateur de Yamabe $4 \frac{n-1}{n-2} \Delta_{g}+s_{g}$ (le fait de considérer la première valeur propre n'intervient que pour s'assurer qu'elle est simple, donc facilement dérivable en tant que fonction de la métrique). La principale différence avec les fonctionnelles considérées plus haut réside dans le fait que $\lambda_{1}$ n'est pas une fonctionnelle locale. On démontre que $d \lambda_{1}=\psi_{g}^{2}$ où $\psi_{g}$ désigne la fonction propre de l'opérateur de Yamabe pour la métrique $g$ associée à cette valeur propre. La relation de conservation qui s'en déduit s'énonce alors comme suit :

Proposition 5. Pour tout champ de vecteurs conforme $X$ et pour toute métrique $g$ de la classe conforme $\Gamma$,

$$
\int_{M} X \cdot \psi_{g}^{2} v_{g}=0
$$

Il est intéressant de noter qu'à la différence de la Proposition 4, dont une démonstration peut être obtenu par une intégration astucieuse à partir de l'identité que vérifie un champ de vecteurs conforme, il ne semble pas facile d'obtenir la relation de la Proposition 5 par un calcul direct. Ce phénomène est probablement dû au caractère global de la fonction $\lambda_{1}$.

Il y a encore une autre famille de 1-formes différentielles fermées sur $\Gamma$ qu'il est particulièrement intéressant de considérer. Cette famille est reliée aux invariants caractéristiques de la variété $M$. Il est bien connu que, par la théorie de Chern-Weil, on peut exprimer ces invariants comme intégrales de polynômes en la courbure de n'importe quelle métrique riemannienne sur $M$.

Ainsi si la dimension de $M$ est $2 k$, il existe un polynôme en la courbure $\chi_{2 k}\left(R^{g}\right)$ tel que la caractéristique d'Euler de $M$, soit $\chi(M)$, s'exprime comme $\chi(M)=\int_{M} \chi_{2 k}\left(R^{g}\right) v_{g}$. Indépendamment de la dimension $n$ de $M$, il est donc naturel de considérer sur $\Gamma$ la forme différentielle que nous notons encore $\chi_{2 k}$ définie par $v_{g} \mapsto \chi_{2 k}\left(R^{g}\right)$. On peut noter que si $n<2 k$, alors pour toute métrique riemannienne $g$ sur $M, \chi_{2 k}\left(R^{g}\right) \equiv 0$.

A cause de sa nature géométrique, la forme $\chi_{2 k}$ est évidemment invariante par le groupe $G$, mais nous avons aussi

Lemme 6. La 1-forme différentielle $\chi_{2 k}$ est fermée sur $\Gamma$. Si $2 k<n, \chi_{2 k}$ est, $\grave{a}$ un coefficient près, la différentielle de la fonction $v_{g} \mapsto \int_{M} \chi_{2 k}\left(R^{g}\right) v_{g}$.

Il est donc possible d'appliquer aux 1-formes différentielles fermées et invariantes $\chi_{2 k}$ le raisonnement de la Section 1 et d'obtenir ainsi de nouveaux invariants intégraux fonctionnels.

THÉORÈme 7. Pour tout champ de vecteurs conforme X et pour toute métrique $g$ de la classe conforme $\Gamma$,

$$
\int_{M} X \cdot \chi_{2 k}\left(R^{g}\right) v_{g}=0 .
$$


On peut noter que la forme $\sigma$ n'est rien d'autre que $\chi_{2}$ de telle sorte que la relation d'intégrabilité de Kazdan-Warner est un cas particulier du Théorème 7 .

Comme nous concentrons notre attention sur une classe conforme et que les nombres de Pontryaguine de $M$ s'expriment avec le tenseur de courbure conforme de Weyl qui est invariant par changement conforme de métrique (quand on lui donne la bonne variance), il ne semble pas possible de tirer d'information intéressante de cette famille.

3. Une nouvelle approche de l'obstruction de Futaki. Dans [4], A. Futaki trouve un nouvel invariant d'une classe de Kähler d'une variété complexe $M$ de dimension complexe $m$, qui présente l'intérêt d'être une obstruction à l'existence de métriques de Kähler-Einstein dans cette classe. Si, pour une métrique kählérienne $\omega$, on désigne par $s_{\omega}$ la courbure et si on introduit la fonction $f_{\omega}$ d'intégrale nulle par rapport à l'élément de volume $\omega^{m}$ telle que $\Delta_{\omega} f_{\omega}=s_{\omega}-s_{\omega}^{0}$ (où $s_{\omega}^{0}$ désigne la moyenne de $s_{\omega}$ ), A. Futaki montre que "pour tout champ de vecteurs holomorphe $X$ sur $M, \int_{M} X \cdot f_{\omega} \omega_{m}$ est indépendant de la forme de Kähler prise dans la classe que l'on considère". Cette intégrale est bien sûr nulle s'il existe une métrique à courbure scalaire constante dans la classe de Kähler, a fortiori s'il existe une métrique de Kähler-Einstein. Il remarque aussi que cette relation est une généralisation directe de la relation d'intégrabilité de Kazdan-Warner.

Cette relation peut être décrite dans le formalisme que nous avons utilisé pour l'étude d'une classe conforme de métriques de la façon suivante. Nous prenons comme espace $\mathcal{O}$ d'objets géométriques une classe de Kähler $K$ que nous identifions à l'ouvert de l'espace des fonctions $\psi$ définies sur $M$ telles que pour une métrique de Kähler $\omega_{0}$ prise comme origine, $\omega_{0}+\partial \bar{\partial} \psi$ est une forme de Kähler. Le groupe $G$ est le groupe des transformations holomorphes de la variété complexe $M$.

Il s'agit maintenant de trouver des formes différentielles fermées et invariantes sur $K$. On peut bien sûr considérer des 1-formes qui se trouvent être différentielles exactes de fonctions invariantes. Dans notre description, un vecteur tangent à $K$ est une fonction définie sur $M$, et un vecteur cotangent n'est rien d'autre qu'une $2 m$-forme différentielle définie sur $M$. Si à une forme de Kähler $\omega$, on associe la $2 m$-forme différentielle $\varrho_{\omega} \wedge \omega^{m-1}$ (où $\varrho_{\omega}$ désigne la forme de Ricci de la métrique kählérienne $\omega$ qui, comme il est bien connu, représente à un coefficient universel près la première classe de Chern de $M$ ), alors on retrouve l'invariant intégral fonctionnel de Futaki. Il faut bien sûr vérifier que cette forme est fermée, car son invariance est automatique à cause de sa nature géométrique et du fait que les transformations holomorphes commutent avec l'opérateur $\partial \bar{\partial}$. L'apparence différente de la relation de Futaki tient au fait que les éléments de l'espace $K$ que nous considérons doivent être dérivés deux fois pour définir les véritables objets géométriques qui importent, à savoir les classes de Kähler.

La fin de calcul se présente donc comme suit. Il s'agit de trouver la forme que prend le champ de vecteurs $\bar{X}$ tangent à l'espace $K$. Pour cela, il suffit de remarquer que la 2 -forme de type $(1,1) \mathcal{L}_{X} \omega$ appartient en chaque point $\psi$ de 
$K$ à l'image de l'opérateur $\partial \bar{\partial}$ et que l'on peut poser $\mathcal{L}_{X}\left(\omega_{0}+\partial \bar{\partial} \psi\right)=\partial \bar{\partial} \psi_{X}$ pour une fonction $\psi_{X}$ que l'on peut prendre d'intégrale nulle. On a alors $\bar{X}(\psi)=$ $\psi_{X}$. Le développement de la Section 1 affirme alors que pour toutes formes de Kähler $\omega$ et $\omega^{\prime}$ appartenant à la même classe et pour tout champ de vecteurs holomorphe $X$,

$$
\int_{M} \psi_{X} \varrho_{\omega} \wedge \omega^{m-1}=\int_{M} \psi_{X}^{\prime} \varrho_{\omega}^{\prime} \wedge \omega^{\prime m-1} .
$$

En décomposant la 2-forme fermée $\varrho_{\omega}$ en sa partie harmonique et sa partie exacte qui se trouve être $\partial \bar{\partial} f_{\omega}$ (où $f_{\omega}$ est la fonction de Futaki), on obtient

$$
\begin{aligned}
\int_{M} \psi_{X} \varrho_{\omega} \wedge \omega^{m-1} & =\int_{M} \psi_{X} s_{\omega}=s_{\omega}^{0} \int_{M} \psi+\int_{M} \psi_{X} \Delta_{\omega} f_{\omega} \omega^{m} \\
& =\int_{M} \Delta_{\omega} \psi_{X} f_{\omega} \omega^{m}=-\int_{M} \operatorname{div}_{\omega} X f_{\omega} \omega^{m}=\int_{M} X \cdot f_{\omega} \omega^{m},
\end{aligned}
$$

ce qui donne la relation trouvée par A. Futaki.

On peut noter que la 1-forme différentielle considérée sur $M$ est, à un coefficient universel près, l'intégrand du nombre caractéristique kählérien obtenu en intégrant sur $M$ le cup-produit de la première classe de Chern par la puissance qu'il faut de la forme de Kähler. La méthode développée permet de trouver des généralisations de la relation de Futaki. Nous ne les exposerons pas ici.

4. Un lien avec les anomalies de jauge? Nous ne ferons que de brefs commentaires sur l'analogie qui semble exister entre les développements que nous venons de faire et les anomalies de jauge considérées par les physiciens.

Rappelons que les physiciens désignent sous le terme d'anomalies certains termes de nature cohomologique apparaissant dans le remplacement d'une fonctionnelle classique par son analogue quantique et qui rendent cette opération non consistante par brisure de symétrie. (La non-nullité de ces termes les amène à rejeter le modèle en question, d'où le terme d'anomalie.) Les espaces considérés sont soit l'espace de toutes les connexions adaptées à un groupe de symétrie (anomalies chirales), soit l'espace des métriques riemanniennes sur une variété (anomalies gravitationnelles). Dans ces situations, il y a un groupe agissant sur ces espaces dit groupe des symétries locales (le groupe de jauge dans le cas des connexions, le groupe des difféomorphismes dans le cas gravitationnel), ce qui nous rapproche du cadre que nous avons développé. De la même façon que pour les deux exemples que nous avons présentés, les espaces considérés sont contractiles et le groupe qui agit est non compact.

La cohomologie considérée par les physiciens n'est pas la cohomologie ordinaire, car ils ne s'intéressent qu'à des objets définis localement sur l'espace de configuration, d'où le nom souvent avancé de cohomologie locale sans qu'une définition mathématique vraiment rigoureuse n'ait été donnée. Les invariants caractéristiques jouent aussi un grand rôle dans les fonctionnelles qu'ils considèrent. 
Malgré des ressemblances frappantes, il est cependant encore difficile d'énoncer des résultats plus précis, décrivant la relation que les invariants intégraux fonctionnels présentés dans cet exposé entretiennent avec les anomalies.

\section{Références}

[1] A. Bahri et J. M. Coron, Une théorie des points critiques à l'infini pour l'équation de Yamabe et le problème de Kazdan-Warner, C. R. Acad. Sci. Paris 300 (1985), 513-516.

[2] J. P. Bourguignon and J. P. Ezin, Scalar curvature functions in a conformal class of metrics and conformal transformations, preprint, Ecole Polytechnique.

[3] J. F. Escobar and R. Schœn, Conformal metrics with prescribed scalar curvature, preprint, Univ. of California, San Diego, 1985.

[4] A. Futaki, An obstruction to the existence of Einstein Kähler metrics, Invent. Math. 73 (1983), 437-443.

[5] J. L. Kazdan and F. Warner, Curvature functions on compact 2-manifolds, Ann. of Math. 99 (1974), 14-47.

[6] J. Lelong-Ferrand, Transformations conformes et quasi-conformes des variétés riemanniennes compactes (démonstration de la conjecture de Lichnerowicz), Acad. Roy. Belg. Cl. Sci. Mémoire 39 (5) (1971).

[7] M. Obata, The conjectures on conformal transformations of Riemannian manifolds, J. Differential Geom. 6 (1971), 247-258. 